PROCEEDINGS OF THE

AMERICAN MATHEMATICAL SOCIETY

Volume 140, Number 12, December 2012, Pages 4097-4103

S 0002-9939(2012)11275-4

Article electronically published on April 10, 2012

\title{
A NOTE ON SIMPLE $a$-POINTS OF $L$-FUNCTIONS
}

\author{
S. M. GONEK, S. J. LESTER, AND M. B. MILINOVICH
}

(Communicated by Matthew A. Papanikolas)

\begin{abstract}
We prove, subject to certain hypotheses, that a positive proportion of the $a$-points of the Riemann zeta-function and Dirichlet $L$-functions with primitive characters are simple and discuss corresponding results for other functions in the Selberg class. We also prove an unconditional result of this type for the $a$-points in fixed strips to the right of the line $\Re s=1 / 2$.
\end{abstract}

\section{INTRODUCTION}

Let $F_{a}(s)=\zeta(s)-a$, where $\zeta(s)$ is the Riemann zeta-function, $s=\sigma+i t$, and $a$ is a nonzero complex number. The zeros of $F_{a}(s)$, which we denote by $\rho_{a}=\beta_{a}+i \gamma_{a}$, are called the $a$-points of $\zeta(s)$, and their distribution has long been an object of study (cf. 6, 9, 11 for the principal results and further references). The resulting value distribution theory is quite beautiful and not as widely known as it should be. Some of the most basic facts are these. First, there exists a number $n_{0}(a)$ such that $F_{a}(s)$ has a zero quite close to $s=-2 n$ for all integers $n \geq n_{0}(a)$, and there are at most finitely many other zeros in $\Re s \leq 0$. (Levinson [6] states this for $\Re s \leq-2$, but it is not difficult to see that it holds for $\Re s \leq 0$.) We call these zeros of $F_{a}(s)$ trivial a-points. The remaining zeros all lie in a strip $0<\Re s<A$, where $A$ depends on $a$, and we call these nontrivial a-points. For these we have

$$
N_{a}(T)=\sum_{\substack{0<\gamma_{a}<T \\ \beta_{a}>0}} 1=\frac{T}{2 \pi} \log \frac{T}{2 \pi}-\frac{T}{2 \pi}+O_{a}(\log T)
$$

if $a \neq 1$; if $a=1$, there is an additional term $-\log 2(T / 2 \pi)$ on the right-hand side of the above equation (cf. 6] or 9]). The zeros of $F_{a}(s)$ cluster near the line $\Re s=1 / 2$ (Levinson [6]), as do the zeros of $\zeta(s)$ (Titchmarsh [1]), of which there are approximately the same number in the strip $0<\Re s<T$ (see (2.1) below). However, $F_{a}(s)$ has $\gg T$ zeros with $\beta_{a}>\sigma$ and $0<\gamma_{a}<T$ for each fixed $\sigma$ with $1 / 2<\sigma \leq 1$, whereas $\zeta(s)$ has at most $O\left(T^{\theta(\sigma)}\right)$ zeros in such a region, where $\theta(\sigma)<1$ (Titchmarsh [11]).

By analogy with the case $a=0$, it is natural to ask whether all or almost all of the $a$-points $\rho_{a}$ are simple. By simple we mean that $F_{a}\left(\rho_{a}\right)=0$, but $F_{a}^{\prime}\left(\rho_{a}\right)=\zeta^{\prime}\left(\rho_{a}\right) \neq$ 0 . The trivial zeros near the numbers $-2 n$ with $n \geq n_{0}(a)$ are known to be simple. Also, for almost every $a$, each $a$-point is simple, for there are an uncountable number

Received by the editors March 18, 2011 and, in revised form, May 18, 2011 and May 24, 2011. 2010 Mathematics Subject Classification. Primary 11M06, 11M26.

Key words and phrases. a-points, simple zeros, Riemann zeta-function, $L$-functions, Selberg class.

Research of the first author was partially supported by NSF grant DMS-0653809.

(C)2012 American Mathematical Society 
of $a$ 's but only a countable number of zeros of $\zeta^{\prime}(s)$. Garunkštis and Steuding [ [5] have recently shown that for every $a$ an infinite number of $a$-points are simple. On the other hand, for some $a$ there certainly are multiple zeros of $F_{a}(s)$ : if $\zeta^{\prime}\left(\rho^{\prime}\right)=0$ and $\zeta\left(\rho^{\prime}\right)=a$, then $\rho^{\prime}$ is an $a$-point of order at least two.

The purpose of this paper is to investigate the density of simple $a$-points both to the left and right of the critical line $\Re s=1 / 2$. Our methods also apply to the $a$-points of a number of other functions in the Selberg class [9, such as Dirichlet $L$-functions with primitive characters, but we primarily focus on the Riemann zetafunction and later indicate the modifications necessary to handle other functions.

\section{2. $a$-POINTS TO THE LEFT OF THE LINE $\Re s=1 / 2$}

Analogously to $N_{a}(T)$ in (1.1), we define $N(T)$ and $N^{\prime}(T)$ to be the number of complex zeros in the half-strip $\sigma>0,0<t<T$ of $\zeta(s)$ and $\zeta^{\prime}(s)$, respectively. Then (see [2, 7])

$$
N(T)=\frac{T}{2 \pi} \log \frac{T}{2 \pi}-\frac{T}{2 \pi}+O(\log T)
$$

and

$$
N^{\prime}(T)=\frac{T}{2 \pi} \log \frac{T}{4 \pi}-\frac{T}{2 \pi}+O(\log T) .
$$

We also let $N_{a}\left(\sigma_{1}, \sigma_{2}, T\right), N\left(\sigma_{1}, \sigma_{2}, T\right)$, and $N^{\prime}\left(\sigma_{1}, \sigma_{2}, T\right)$ denote the number of $a$-points of $\zeta(s)$, zeros of $\zeta(s)$, and zeros of $\zeta^{\prime}(s)$, respectively, with real part in $\left(\sigma_{1}, \sigma_{2}\right)$ and imaginary part in $(0, T)$.

We first consider the question of simple $a$-points under the assumption that the Riemann Hypothesis is true. By a theorem of Speiser [10, the Riemann Hypothesis implies that $\zeta^{\prime}(s)$ has no zeros in the strip $0<\sigma<1 / 2$. Furthermore, Selberg [9] (see also Tsang [12]) has shown that on the Riemann Hypothesis at least 1/2 of the nontrivial $a$-points lie to the left of $\Re s=1 / 2$. It follows that these must all be simple. Thus, we easily have

Theorem 1. Assume the Riemann Hypothesis. Then at least

$$
\left(\frac{1}{2}+o(1)\right) \frac{T}{2 \pi} \log \frac{T}{2 \pi}
$$

of the nontrivial a-points of $\zeta(s)$ with ordinates in $(0, T)$ are simple and lie to the left of the line $\Re s=1 / 2$.

It was pointed out by Selberg that independently of the Riemann Hypothesis, one can show that if $a=|a| e^{i \theta} \neq 0$ for almost all $\theta \in[0,2 \pi)$, then at least half the $a$-points lie to the left of $\Re s=1 / 2$.

Next we estimate the number of simple $a$-points under another hypothesis. In [9] Selberg says it is likely that for a given $a \neq 0$, three quarters of the $a$-points are to the left of the line $\Re s=1 / 2$ (see Tsang [12] and Montgomery's review 1 of [6]). We state this as

Conjecture 1 (Selberg's $a$-Point Conjecture). For all $a \neq 0$ we have

$$
N_{a}(0,1 / 2, T)=\left(\frac{3}{4}+o(1)\right) \frac{T}{2 \pi} \log \frac{T}{2 \pi} .
$$

\footnotetext{
${ }^{1}$ MathSciNet: MR0406952(53\#10737)
} 
To support the conjecture, Selberg notes that if the ratio of two complex numbers $a$ and $a^{\prime}$ is negative, one can show that $3 / 4$ of the zeros of $(\zeta(s)-a)\left(\zeta(s)-a^{\prime}\right)$ lie to the left of $\Re s=1 / 2$ for almost all values of the argument of $a$.

Now let $N^{(0)}(T)$ denote the number of zeros of $\zeta(s)$ with real part equal to $1 / 2$ and imaginary part in $(0, T)$, and set

$$
\theta=\liminf _{T \rightarrow \infty} \frac{N^{(0)}(T)}{N(T)} .
$$

Our next theorem asserts that if Selberg's $a$-Point Conjecture is true and enough zeros of $\zeta(s)$ are on the line $\Re s=1 / 2$, then a positive proportion of the $a$-points are still simple.

Theorem 2. Assume Selberg's a-Point Conjecture and let $\theta$ be as in (2.3). Then at least

$$
\left(\theta-\frac{1}{4}+o(1)\right) \frac{T}{2 \pi} \log \frac{T}{2 \pi}
$$

of the nontrivial a-points of $\zeta(s)$ with ordinates in $(0, T)$ are simple and lie to the left of the line $\Re s=1 / 2$.

Proof. Levinson and Montgomery 7 have shown unconditionally that

$$
N^{\prime}(0,1 / 2, T)=N(0,1 / 2, T)+O(\log T) .
$$

By the functional equation for $\zeta(s)$, the nontrivial zeros of $\zeta(s)$ are symmetric about the line $\Re s=1 / 2$. Thus, at most $(1-\theta) / 2$ of these zeros lie to the left of $\Re s=1 / 2$, and at most $(1-\theta) / 2$ lie to the right of it. By (2.1), (2.2), and (2.4), it therefore follows that

$$
N^{\prime}(0,1 / 2, T) \leq\left(\frac{1-\theta}{2}+o(1)\right) \frac{T}{2 \pi} \log \frac{T}{2 \pi} .
$$

For $z \in \mathbb{C}$, let $m_{a}(z)=0$ if $z$ is not an $a$-point of $\zeta(s)$; if $z$ is an $a$-point, let $m_{a}(z)$ denote its multiplicity as an $a$-point. Similarly, let $m^{\prime}(z)=0$ if $z$ is not a zero of $\zeta^{\prime}(s)$, and, if it is, let $m^{\prime}(z)$ denote its multiplicity. Then when $z=\rho_{a}$ is an $a$-point, we clearly have $m^{\prime}\left(\rho_{a}\right)=m_{a}\left(\rho_{a}\right)-1$. Let $\mathcal{R}(T)$ denote the rectangular region $(0,1 / 2) \times(0, T) \subset \mathbb{C}$. Then

$$
\begin{aligned}
\sum_{\substack{\rho_{a} \in \mathcal{R}(T) \\
m_{a}\left(\rho_{a}\right) \geq 2}} m_{a}\left(\rho_{a}\right) & =\sum_{\substack{\rho_{a} \in \mathcal{R}(T) \\
m^{\prime}\left(\rho_{a}\right) \geq 1}}\left(m^{\prime}\left(\rho_{a}\right)+1\right) \\
\leq 2 & \sum_{\substack{\rho_{a} \in \mathcal{R}(T) \\
m^{\prime}\left(\rho_{a}\right) \geq 1}} m^{\prime}\left(\rho_{a}\right) \\
\leq 2 & \sum_{\substack{\rho^{\prime} \in \mathcal{R}(T) \\
m^{\prime}\left(\rho^{\prime}\right) \geq 1}} m^{\prime}\left(\rho^{\prime}\right)=2 N^{\prime}(0,1 / 2, T) .
\end{aligned}
$$

Here and below our sums are over distinct $a$-points $\rho_{a}$ and zeros $\rho^{\prime}$ if $\zeta^{\prime}(s)$. We also have

$$
N_{a}(0,1 / 2, T)=\sum_{\substack{\rho_{a} \in \mathcal{R}(T) \\ m_{a}\left(\rho_{a}\right)=1}} 1+\sum_{\substack{\rho_{a} \in \mathcal{R}(T) \\ m_{a}\left(\rho_{a}\right) \geq 2}} m_{a}\left(\rho_{a}\right)
$$


Combining this with (2.6), we find that the number of simple a-points in $\mathcal{R}(T)$ satisfies

$$
\sum_{\substack{\rho_{a} \in \mathcal{R}(T) \\ m_{a}\left(\rho_{a}\right)=1}} 1 \geq N_{a}(0,1 / 2, T)-2 N^{\prime}(0,1 / 2, T) .
$$

Thus, by (2.5) and Conjecture 1 we have

$$
\sum_{\substack{\rho_{a} \in \mathcal{R}(T) \\ m_{a}\left(\rho_{a}\right)=1}} 1 \geq\left(\frac{3}{4}-(1-\theta)+o(1)\right) \frac{T}{2 \pi} \log \frac{T}{2 \pi}=\left(\theta-\frac{1}{4}+o(1)\right) \frac{T}{2 \pi} \log \frac{T}{2 \pi} .
$$

This completes the proof of the theorem.

Bui et al. 4 have recently shown that $\theta \geq .4105$. Using this in Theorem 2, we obtain

Corollary 1. Assume Selberg's a-Point Conjecture. Then at least

$$
\left(\frac{4}{25}+o(1)\right) \frac{T}{2 \pi} \log \frac{T}{2 \pi}
$$

of the nontrivial a-points of $\zeta(s)$ with ordinates in $(0, T)$ are simple and lie to the left of the line $\Re s=1 / 2$.

We state the case $\theta=1$ of Theorem 2 separately as

Corollary 2. Assume Selberg's a-Point Conjecture and that 100 percent of the zeros of $\zeta(s)$ lie on the line $\Re s=1 / 2$. Then at least

$$
\left(\frac{3}{4}+o(1)\right) \frac{T}{2 \pi} \log \frac{T}{2 \pi}
$$

of the nontrivial a-points of $\zeta(s)$ with ordinates in $(0, T)$ are simple and lie to the left of the line $\Re s=1 / 2$.

In particular, the conclusion of Corollary 2 holds under the assumption of Selberg's $a$-Point Conjecture and the Riemann Hypothesis.

Our arguments extend to Dirichlet $L$-functions with a fixed primitive character $\chi$ as well. To see this we note the following:

(i) $L(s, \chi)$ and $F_{a}(s, \chi)=L(s, \chi)-a$ both have $\sim(T / 2 \pi) \log T / 2 \pi$ nontrivial zeros with real part greater than 0 and imaginary part in $(0, T)$.

(ii) Levinson and Montgomery's result that $\zeta(s)$ and $\zeta^{\prime}(s)$ have the same number of zeros to within $O(\log T)$ in the rectangular region $\mathcal{R}(T)$ holds for $L(s, \chi)$ and $L^{\prime}(s, \chi)$ (see Powell [8]).

(iii) Selberg's result that at least $1 / 2$ of the $a$-points of the zeta-function lie to the left of the line $\Re s=1 / 2$ is true for $L(s, \chi)$ as well, provided the Riemann Hypothesis is true for $L(s, \chi)$ (see Selberg [9]).

(iv) Selberg actually asserts the $a$-Point Conjecture for every primitive function in the Selberg class, so in particular for $L(s, \chi)$. 
It follows from (i)-(iv) that the analogues of Theorems 1 and 2 are true for any Dirichlet $L$-function $L(s, \chi)$ with a primitive character $\chi$. We state this as

Theorem 3. Let $L(s, \chi)$ denote a Dirichlet $L$-function with a primitive character $\chi$. If the Riemann Hypothesis is true for $L(s, \chi)$, then at least

$$
\left(\frac{1}{2}+o(1)\right) \frac{T}{2 \pi} \log \frac{T}{2 \pi}
$$

of the nontrivial a-points of $L(s, \chi)$ with ordinates in $(0, T)$ are simple and lie to the left of the line $\Re s=1 / 2$. If instead of the Riemann Hypothesis we assume that Selberg's a-Point Conjecture holds for $L(s, \chi)$, and if $\theta_{\chi}$ is the analogue for $L(s, \chi)$ of $\theta$ (cf. (2.3) $)$, then at least

$$
\left(\theta_{\chi}-\frac{1}{4}+o(1)\right) \frac{T}{2 \pi} \log \frac{T}{2 \pi}
$$

of the nontrivial a-points of $L(s, \chi)$ with ordinates in $(0, T)$ are simple and lie to the left of the line $\Re s=1 / 2$.

Bauer [1] has shown that $\theta_{\chi} \geq .365815$ for primitive Dirichlet $L$-functions. Using this in the second part of Theorem 3 , we obtain

Corollary 3. Assume Selberg's a-Point Conjecture holds for a Dirichlet L-function $L(s, \chi)$ with $\chi$ a primitive character. Then at least

$$
(.1158+o(1)) \frac{T}{2 \pi} \log \frac{T}{2 \pi}
$$

of the nontrivial a-points of $L(s, \chi)$ with ordinates in $(0, T)$ are simple and lie to the left of the line $\Re s=1 / 2$.

Of course, the result corresponding to Corollary 2 holds for $L(s, \chi)$ as well.

Analogues of (i) and (iii) above are known for all functions in the Selberg class. We have not found the analogue of (ii) for the Selberg class in the literature, but it is likely that it can be proved by making minor modifications to the argument of Levinson and Montgomery in [7. If that is the case and the Riemann Hypothesis is true for the functions of the Selberg class, then at least $1 / 2$ of their $a$-points are simple. Furthermore, if the analogue of Selberg's $a$-Point Conjecture also holds, then $3 / 4$ of the $a$-points are simple.

\section{3. $a$-POINTS TO THE RIGHT OF THE LINE $\Re s=1 / 2$}

Let $\sigma_{1}$ and $\sigma_{2}$ be fixed with $1 / 2<\sigma_{1}<\sigma_{2}<1$, and recall that $N_{a}\left(\sigma_{1}, \sigma_{2}, T\right)$ is the number of $a$-points of $\zeta(s)$ in the rectangular region $\left(\sigma_{1}, \sigma_{2}\right) \times(0, T)$. Then there is a positive constant $C_{a}\left(\sigma_{1}, \sigma_{2}\right)$ such that

$$
N_{a}\left(\sigma_{1}, \sigma_{2}, T\right) \sim C_{a}\left(\sigma_{1}, \sigma_{2}\right) T
$$

(see Borchsenius and Jessen [3], Theorem 14). Thus a natural question is whether a positive proportion of these $a$-points are simple. In this section we show unconditionally that this is indeed the case. The proof uses Voronin's universality theorem for the Riemann zeta-function [13]. 
Theorem 4. Let $1 / 2<\sigma_{1}<\sigma_{2}<1$ with $\sigma_{1}, \sigma_{2}$ fixed, and let $a \neq 0$. Then there is a positive constant $c_{a}\left(\sigma_{1}, \sigma_{2}\right)$ such that at least $c_{a}\left(\sigma_{1}, \sigma_{2}\right) T$ of the a-points of $\zeta(s)$ in the region $\left(\sigma_{1}, \sigma_{2}\right) \times(0, T)$ are simple.

Proof. Set $s_{0}=\left(\sigma_{1}+\sigma_{2}\right) / 2$, let $0<R<\left(\sigma_{2}-\sigma_{1}\right) / 2<1 / 4$, and let $D_{R}$ be the closed disc of radius $R$ centered at $s_{0}$. Consider the function

$$
f(s)=a+\frac{|a|}{2}\left(s-s_{0}\right)
$$

on $D_{R}$. By Voronin's universality theorem (see [13]), for any $\epsilon>0$ there is a sequence $\left\{\tau_{n}\right\}_{n=1}^{\infty}$ such that the discs $D_{R}+i \tau_{n}$ are nonoverlapping,

$$
\left|\left\{\tau_{n}: 0<\tau_{n} \leq T\right\}\right| \gg T,
$$

and

$$
\max _{s \in \partial D_{R}}\left|\zeta\left(s+i \tau_{n}\right)-f(s)\right|<\epsilon
$$

for all $n$. By our choice of $f(s)$ we also have

$$
\min _{s \in \partial D_{R}}|f(s)-a|=\frac{|a| R}{2} .
$$

Taking $\epsilon=|a| R^{2} / 100$, we see that $\epsilon<|a| R / 2$, since $R<1 / 4$. Thus, by Rouché's Theorem, $\zeta\left(s+i \tau_{n}\right)-a$ and $f(s)-a$ have the same number of zeros inside $D_{R}$ for each $n$, namely one counting multiplicities. It follows that the zero of $\zeta\left(s+i \tau_{n}\right)-a$ is a simple $a$-point of $\zeta(s)$. Therefore, there are $\gg T$ simple $a$-points of $\zeta(s)$ in $\left(\sigma_{1}, \sigma_{2}\right) \times(0, T)$. This completes the proof.

Analogues of Voronin's Universality Theorem are known to hold for many functions in the Selberg class and, in particular, for Dirichlet $L$-functions. The above proof therefore extends to them.

Our theorems leave open the question of the simplicity of $a$-points on or slightly to the right of the line $\Re s=1 / 2$. It is not difficult to show that if $\zeta^{\prime}\left(\frac{1}{2}+i t_{0}\right)=0$, then $\zeta\left(\frac{1}{2}+i t_{0}\right)=0$. Hence, every $a$-point of $\zeta(s)$ on the line $\Re s=1 / 2$ is simple. However, as Selberg suggests (see [9, p. 58, footnote 10), there are probably only finitely many such $a$-points. This together with Selberg's $a$-Point Conjecture implies that $(1 / 4+o(1))(T /(2 \pi)) \log T /(2 \pi)$ of the $a$-points of $\zeta(s)$ lie to the right of $\Re s=1 / 2$. We conjecture that almost all of these are simple too. It would be interesting if one could show that a positive proportion of them are.

\section{REFERENCES}

1. P. J. Bauer, Zeros of Dirichlet L-series on the critical line, Acta Arith. 93 (2000), 37-52. MR 1760087 (2001h:11113)

2. B. C. Berndt, The number of zeros for $\zeta^{(k)}(s)$, J. London Math. Soc. (2) 2 (1970), 577-580. MR0266874 (42:1776)

3. V. Borchsenius and B. Jessen, Mean motions and values of the Riemann zeta function, Acta Math. 80 (1948), 97-166. MR0027796 (10:356b)

4. H. M. Bui, J. B. Conrey, and M. P. Young, More than $41 \%$ of the zeros of the zeta function are on the critical line, to appear in Acta Arith. Available on the arXiv at http://arxiv.org/pdf/1002.4127v2.

5 . R. Garunkštis and J. Steuding, On the roots of the equation $\zeta(s)=a$, preprint. Available on the arXiv at http://arxiv.org/abs/1011.5339v1.

6. N. Levinson, Almost all roots of $\zeta(s)=a$ are arbitrarily close to $\sigma=1 / 2$, Proc. Nat. Acad. Sci. USA 72 (1975), 1322-1324. MR0406952 (53:10737) 
7. N. Levinson and H. L. Montgomery, Zeros of the derivatives of the Riemann zeta-function, Acta Math. 133 (1974), 49-65. MR0417074 (54:5135)

8. K. Powell, Topics in analytic number theory, master's thesis, Brigham Young University, Provo, Utah, 2009.

9. A. Selberg, Old and new conjectures and results about a class of Dirichlet series, in: Proceedings of the Amalfi Conference on Analytic Number Theory, Maiori 1989, E. Bombieri et al. (eds.), Università di Salerno, 1992, 367-385. MR.1220477 (94f:11085)

10. A. Speiser, Geometrisches zur Riemannschen Zeta Funktion, Math. Ann. 110 (1934), 514521.

11. E. C. Titchmarsh, The theory of the Riemann zeta-function, 2nd Edition, revised by D. R. Heath-Brown, Oxford University Press, 1986. MR882550 (88c:11049)

12. K. M. Tsang, The distribution of the values of the Riemann zeta-function, Ph.D. dissertation, Princeton Univ., Princeton, NJ, 1984. MR2633927

13. S. M. Voronin, Theorem on the "universality" of the Riemann zeta-function, Izv. Akad. Nauk SSSR Ser. Mat. 39 (1975), 475-486. See also: Math. USSR Izvestija 9 (1975), 443453. MR0472727 (57:12419)

Department of Mathematics, University of Rochester, Rochester, New York 14627

E-mail address: gonek@math.rochester.edu

Department of Mathematics, University of Rochester, Rochester, New York 14627

E-mail address: lester@math.rochester.edu

Department of Mathematics, University of Mississippi, University, Mississippi 38677

E-mail address: mbmilino@olemiss.edu 